\title{
Hierarchical Fingertip Space: A Unified Framework for Grasp Planning and In-Hand Grasp Adaptation
}

\author{
Kaiyu Hang, Miao Li, Johannes A. Stork, Yasemin Bekiroglu, Florian T. Pokorny, \\ Aude Billard and Danica Kragic
}

\begin{abstract}
We present a unified framework for grasp planning and in-hand grasp adaptation using visual, tactile and proprioceptive feedback. The main objective of the proposed framework is to enable fingertip grasping by addressing problems of changed weight of the object, slippage and external disturbances. For this purpose, we introduce the Hierarchical Fingertip Space (HFTS) as a representation enabling optimization for both efficient grasp synthesis and online finger gaiting. Grasp synthesis is followed by a grasp adaptation step that consists of both grasp force adaptation through impedance control and regrasping/finger gaiting when the former is not sufficient. Experimental evaluation is conducted on an Allegro hand mounted on a Kuka LWR arm.
\end{abstract}

Index Terms-Fingertip grasping, Hierarchical Fingertip Space, grasp synthesis, grasp adaptation

\section{INTRODUCTION}

G RASP planning and in-hand grasp adaptation are two complex problems that have commonly been studied separately. Lots of contributions to these problems have been made during the past two decades considering stability modeling and estimation, task based grasping, object representation, grasping synergies and grasp adaptation [1] $-[10]$.

In this paper, we present a framework for fingertip grasping considering an integrated approach to grasp planning and inhand grasp adaptation. The main objective of the framework is to address the problem of grasp instability due to problems such as changed weight of the object, e.g., a container to be filled during grasping, slippage or external disturbances caused by collisions. The framework integrates our previous work of Hierarchical Fingertip Space (HFTS) [11] and grasp adaptation [9], and provides efficient grasp synthesis, grasp force adaptation through impedance control and regrasping/finger gaiting when the former is not sufficient. The approach consists of i) a pre-grasping phase executing grasp synthesis on an efficient representation including both object and hand properties, ii) grasp execution, and iii) a post-grasping phase where tactile feedback and experiences are used for in-hand grasp adaptation, see Fig. 1 and Fig. 2

In the pre-grasping phase, grasp synthesis is formulated as a combinatorial optimization problem considering grasp stability, contact locations and finger gaiting in an integrated

K. Hang, J. A. Stork, Y. Bekiroglu, F. T. Pokorny and D. Kragic are with the Computer Vision and Active Perception Lab, CAS, CSC at KTH Royal Institute of Technology, Stockholm, Sweden. \{kaiyuh, jastork, yaseminb, fpokorny, dani\}@kth.se.

M. Li and A. Billard are with the Learning Algorithms and Systems Laboratory (LASA) at École Polytechnique Fédérale de Lausanne (EPFL), Switzerland \{miao.li, aude.billard\}@epfl.ch.

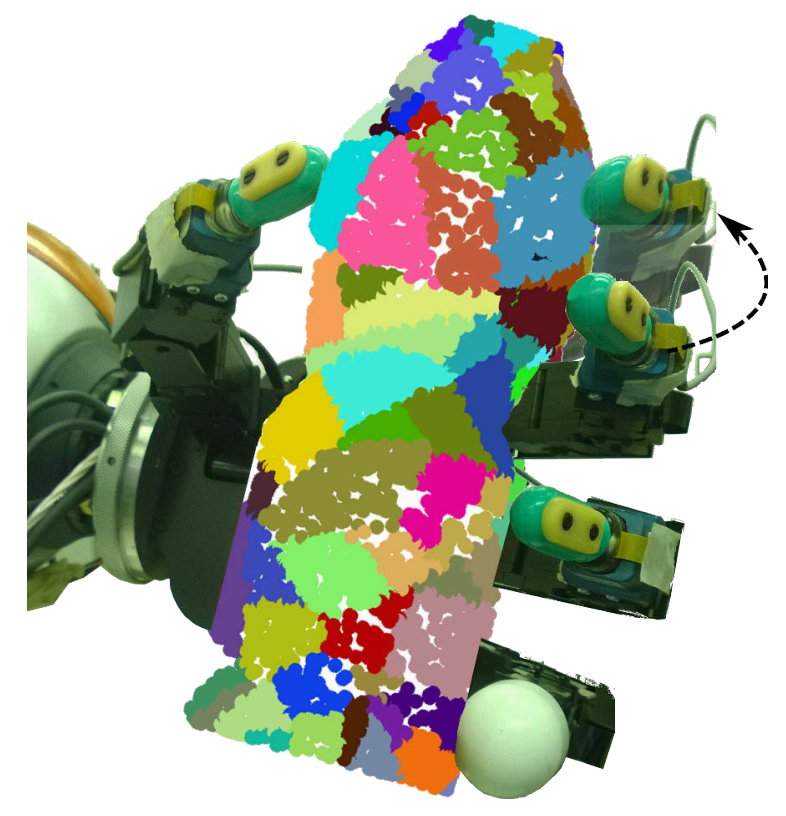

Fig. 1. A visualization of the proposed Hierarchical Fingertip Space concept: Initial fingertip locations are determined by optimizing grasp stability and adaptability using a hierarchical discretization of the object surface and an impedance controller is used to balance grasping forces. If a large disturbance occurs, the grasp is adapted by fingertip gaiting to maintain grasp stability. The new fingertip location is computed using an optimization in the HFTS.

manner. In the post-grasping phase, tactile feedback provides information of the stability of the executed grasp. An offline learned probabilistic model is used to assess the grasp stability and initiate an adaptation of grasp forces, followed by finger gaiting if needed. To the best of our knowledge, this is so far the first system that accomplishes grasp synthesis, stability estimation, online replanning and in-hand adaptation in a unified framework.

Compared to the state of the art and our previous work in [11] and [9], our integrated system:

- provides an optimization framework for both grasp synthesis and finger gaiting;

- closes the loop between grasp planning and control through stability estimation and finger gaiting;

- optimizes grasp adaptability and demonstrates informed finger gaiting optimization by considering viable hand configurations and object shape knowledge.

We review the related work in Sec. III and present the methodology in Sec. III - Sec. V. We evaluate in Sec. VI and then conclude in Sec. VII 

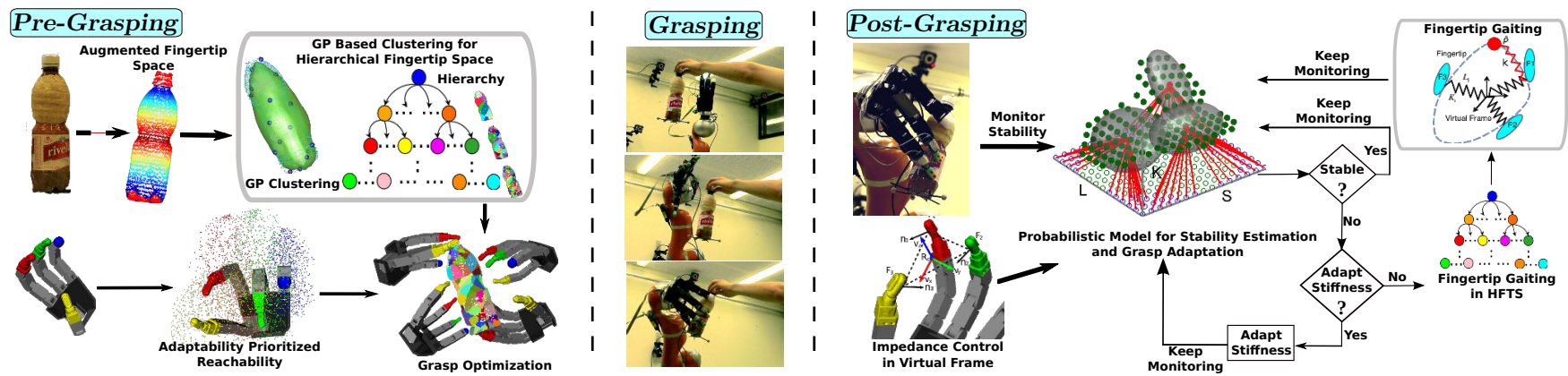

Fig. 2. Schematic overview of the system - Pre-grasping: After the Hierarchical Fingertip Space is generated by a Gaussian Process (GP) based filter, grasps are synthesized by a multi-level refinement strategy. Grasping: The synthesized hand configuration is used to execute the grasp. Post-grasping: Once tactile feedback is available, grasp stability is monitored by a learned probabilistic model. If a grasp is estimated as unstable, the stability is maintained through force adaptation or finger gaiting.

\section{RELATED WORK}

The area of robotic grasping includes problems such as grasp stability analysis, grasp synthesis and hand kinematics, object and task representation, grasp adaptation [6], [10], [12] etc. Although each of these have been studied extensively during the past couple of decades there are rather few systems that have addressed grasp synthesis and in-hand grasp adaptation in an integrated manner.

In terms of object representation for grasping, there are many examples of works that rely on encoding shape properties of objects: Reeb Graph [13], Medial Axis [14], [15], hierarchical box decomposition [16], super-quadrics [17]-[20]. More recent work demonstrates topological analysis of shape for grasping and caging [21], [22]. Our Hierarchical Fingertip Space (HFTS) proposes a method for shape representation that encodes both the global and local geometric properties of the object.

Classical works formulate contact-level grasp synthesis as an optimization problem [8], [12], [23]-[27] for which the objective - grasp stability - is commonly measured using force analysis in the contact wrench space [28]. The problem of calculating feasible hand configurations has also been addressed in this context [2], [29]. To account for uncertainties in physical properties of objects, grasp friction sensitivity [30] and independent contact regions [31] have been investigated. Our approach formulates fingertip grasping as an optimization problem considering grasp stability, adaptability and hand reachability to prepare a grasp for future adaptive execution against physical uncertainties.

Approaches to force based grasp control range from geometry based analytic methods [32]-[34] to learning-based frameworks for force optimization [35], [36]. In-hand manipulation has been addressed as finger gaiting with a rolling contact model and quasi-static assumption [37], [38]. Hybrid position and force control has also been studied [39]-[42] as well as impedance control [43]-[46]. Our approach allows for grasp stabilization through both contact force adaptation and finger gaiting planned in real-time using tactile feedback and the proposed Hierarchical Fingertip Space.

In realistic tasks, the ability to maintain a stable grasp on an object is an integral property of robust systems. A grasp that is originally stable may be perturbed while performing a manipulation with the held object. This is also valid for cases where some properties of the object change - weight can change if a glass held by the robot gets filled, environmental changes can affect friction coefficients, collision may cause slippage, etc. In addition, many of these properties may not be precisely known to start with. Thus, in-hand grasp adaptation may be needed after a grasp has been applied on an object. For this purpose, relying on visual feedback is not sufficient and many of the recent approaches facilitate haptic and proprioceptive information [7], [47]-[53]. Finger gaiting may be further required when applying higher grasping force does not suffice [9], [54]. Our work here builds upon [9], [54] and additionally allows for replanning during grasp execution.

\section{HierarchicAl Fingertip SpaCe AND GRASP OPTIMIZATION}

We start by providing a list of notations used in the paper:

\begin{tabular}{c|c}
\hline \hline $\mathcal{P} \subset \mathbb{R}^{3}$ & An object's point cloud \\
\hline$C_{g}=\left\{c_{1}, \ldots, c_{m} \mid c_{i} \in \mathcal{P}\right\}$ & A grasp defined by $m$ contacts \\
\hline $\mathcal{J}_{g} \in \mathbb{R}^{d}$ & A hand configuration with $d$ DoFs \\
\hline$\Phi(\mathcal{P})=\left\{\phi_{1}, \ldots, \phi_{n_{f}}\right\} \subset \mathcal{P}$ & Fingertip Space built from $\mathcal{P}$ \\
\hline$\phi_{i} \in \Phi(\mathcal{P})$ & A Fingertip Unit \\
\hline$w_{i} \in \mathbb{R}$ & Penalty factor assigned to $\phi_{i}$ \\
\hline$G_{\Phi}=\left(E_{\Phi}, V_{\Phi}\right)$ & A hierarchy of surrogates of $\Phi$ \\
\hline$h o p\left(\phi_{i, j}, \phi_{i, k}\right)$ & Hop distance between nodes in $G_{\Phi}$ \\
\hline$\left(G_{\Phi}\right)_{i}=\left(\left(V_{\Phi}\right)_{i},\left(E_{\Phi}\right)_{i}\right)$ & The $i$-th surrogate approximation of $\Phi$ \\
\hline$w_{i, j} \in \mathbb{R}$ & Penalty factor assigned to a node in $G_{\Phi}$ \\
\hline$\Lambda_{\Phi}=\left(V_{\Lambda}, E_{\Lambda}\right)$ & Hierarchical Fingertip Space \\
\hline$\left(\Lambda_{\Phi}\right)_{i}=\left(\left(V_{\Lambda}\right)_{i},\left(E_{\Lambda}\right)_{i}\right)$ & The $i$-th surrogate approximation of $\Lambda_{\Phi}$ \\
\hline$\lambda_{g} \in E_{\Lambda}$ & A node representing $m$ contacts in $\Lambda_{\phi}$ \\
\hline$g=\left(\lambda_{g}, \mathcal{J}_{g}\right)$ & A grasp defined by $\lambda_{g}$ and $\mathcal{J}_{g}$ \\
\hline$Q\left(\lambda_{g}\right) \in \mathbb{R}$ & Grasp quality defined in 28 \\
\hline$R\left(\lambda_{g}\right) \in \mathbb{R}$ & Grasp reachability residual \\
\hline$A\left(\mathcal{J}_{g}\right) \in \mathbb{R}$ & Grasp adaptability \\
\hline$\theta(g) \in \mathbb{R}$ & Grasp synthesis objective function \\
\hline$R_{o} \in \mathrm{SO}(3)$ & Virtual frame for grasp impedance control \\
\hline$\hat{g}=(K, L, S)$ & A grasp with stiffness $K$, rest length $L$ \\
& and tactile readings $S$ \\
\hline$\Theta$ & Gaussian Mixture Model learned over \\
\hline$\theta^{*}\left(\lambda_{g}\right) \in \mathbb{R}$ & Optimization objective for fingertip relocation \\
\hline \hline
\end{tabular}

TABLE I

LIST OF NOTATIONS USED IN THE PAPER

In the pre-grasping phase, we formulate fingertip grasp synthesis as an optimization problem considering each object represented as a point cloud $\mathcal{P}=\left\{p_{i} \in \mathbb{R}^{3} \mid i \in\left\{1, \ldots, n_{p}\right\}\right\}$. 
We seek $m$ contact locations, $C_{g}=\left\{c_{1}, \ldots, c_{m} \mid c_{i} \in \mathcal{P}\right\}$, on the object surface and a hand configuration, $\mathcal{J}_{g} \in \mathbb{R}^{d}$ where $d$ are controlled joint angles.

We define two concepts: Fingertip Space and Hierarchical Fingertip Space (HFTS). Fingertip Space represents a finite set of contacts on an object surface that are locally flat and large enough for a fingertip [11]. We denote the Fingertip Space as $\Phi(\mathcal{P})=\left\{\phi_{1}, \ldots, \phi_{n_{f}}\right\} \subset \mathcal{P}$ and an element of this set $\phi_{i}$ is called a Fingertip Unit. Fingertip Space $\Phi(\mathcal{P})$ is parametrized by locations and normals of Fingertip Units. We extract the $\Phi(\mathcal{P})$ from $\mathcal{P}$ based on the surface curvature estimated from a set of points $N^{r}\left(p_{i}\right) \subset \mathcal{P}$ within one fingertip size $r$, around a potential contact $p_{i}$. The fingertip space of $\mathcal{P}$ is given by

$$
\Phi(\mathcal{P})=\left\{\phi_{i} \mid \mathcal{K}\left(N^{r}\left(\phi_{i}\right)\right) \leq \kappa, \phi_{i} \in \mathcal{P}\right\}
$$

where $\mathcal{K}\left(N^{r}\left(p_{i}\right)\right)$ is the local surface curvature estimated from $N^{r}\left(p_{i}\right)$ and $\kappa \in \mathbb{R}$ is the empirically determined curvature threshold. In the rest of this paper, we write $\Phi$ instead of $\Phi(\mathcal{P})$. Fig. 3 (left) shows an example of Fingertip Space. To
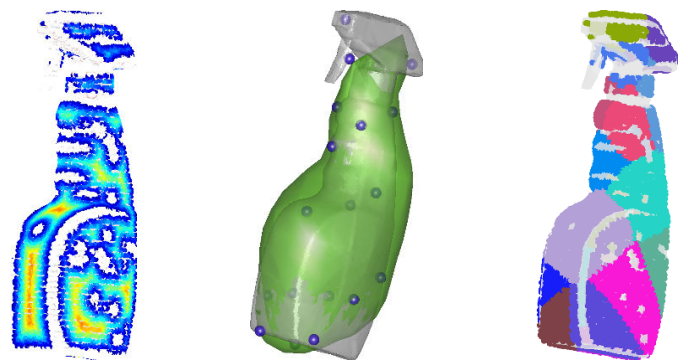

Fig. 3. Left: Fingertip Space with attached penalties rendered by jet colormap, in white are points that have been filtered out by Eq. (1). Note that there is perception noise in planar areas. Middle: GP for the spray model represented by 20 cluster centers. Right: partitions of fingertip units rendered with different colors.

enable finger gaiting, we want our Fingertip Space to encode the space around each Fingertip Unit in an efficient manner. To achieve this, we put a penalty term on admissible regions using a logistic function. Let $c\left(\phi_{i}\right) \in \mathcal{P} \backslash \Phi$ be the closest point to Fingertip Unit $\phi_{i}$ that has been rejected by Eq. (1), the penalty $w_{i}$ for $\phi_{i}$ is computed as:

$$
w_{i}=\frac{1}{1+e^{-\gamma\left\|\phi_{i}-c\left(\phi_{i}\right)\right\|}}
$$

where $\gamma \in \mathbb{R}^{+}$is an elasticity factor, see Fig. 3(left).

\section{A. Multilevel refinement of Fingertip Space}

Given the large number of Fingertip Units per object, formalizing grasp optimization on all combinations of these is computationally impractical. A feasible strategy is to apply Surrogate Models or multilevel refinement [55], [56], that recursively approximate the original optimization problem in a hierarchy of simpler, more tractable problems i.e. surrogate models. We first explain a representation for single fingertip contact optimization and then continue with the definition of Hierarchical Fingertip Space for multiple fingertip contacts.

\footnotetext{
${ }^{1}$ For the SynTouch sensor used in this work, the fingertip size $r$ is $14 \mathrm{~mm}$, http://www.syntouchllc.com/
}

Surrogate approximation of $\Phi$ is constructed by recursively grouping Fingertip Units by cluster analysis using geometric properties. For the optimization of a single contact in $\Phi$ we construct a hierarchy of surrogate approximations of $\Phi$ (see Fig. (4) as a similarity-based graph $G_{\Phi}=\left(E_{\Phi}, V_{\Phi}\right)$, with the hierarchy levels $i \in\{0, \ldots, l-1\}$ representing different scales of surrogate approximations. $\Phi$ is recursively partitioned into smaller sets of fingertip units, denoted as $\hat{\phi}_{i, j} \subset \Phi$, and is represented as a node $\phi_{i, j} \in V_{\Phi}$ in graph $G_{\Phi}$, where $i$ is the level of $\phi_{i, j}$ in the hierarchy and $j$ is the index of the partition in level $i$. We partition the set $\Phi$ in a top-down manner, with parent $\phi_{i, j}$ nodes split into children nodes if $\left|\hat{\phi}_{i, j}\right|>1$. Ultimately, the bottom level of $G_{\Phi}$ consists of nodes representing single fingertip units, $\left|\hat{\phi}_{0, j}\right|=1$. Experimentally and as shown in Fig. 4 , the number of partitioning centers for the level $l-2$ is set to 20 and in the remaining levels to 4 similar to [8].

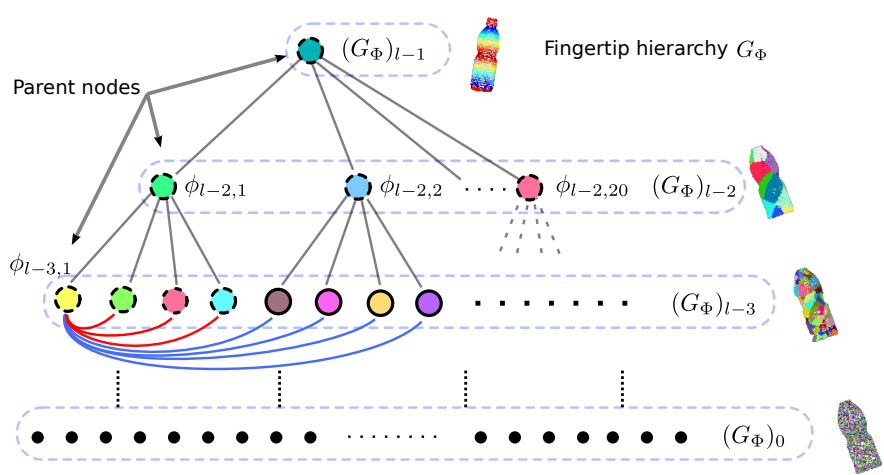

Fig. 4. Surrogate models represented as a graph. Fingertip unit partitions are represented as nodes in different levels of the hierarchy and the connectivities in this graph are represented by edges. Extra connectivities defined by Eq. (3) are exemplified in level $l-3$ for $\phi_{l-3,1}$, with red edges for 2 hops and blue edges for 4 hops.

In the process described above, we require a method for cluster analysis, that fulfills the following properties: $a$ ) The method must be able to group Fingertip Units according to relevant geometric properties. In more detail, the employed similarity measure has to be based on the grasp relevant properties. For point contact with friction, this is captured by position and normal information [23]. b) The recursive grouping in each hierarchy level must result in partitions having similar variance in relevant geometric properties. c) The individual clusters should correspond to connected and compact surface areas such that their average elements represent possible contact locations. These requirements may initially be violated on higher levels but they become increasingly important for lower hierarchy levels. Any clustering method that fulfills these requirement can be employed to construct $G_{\Phi}$, e.g., in our earlier work, we refer to Agglomerative Hierarchical Clustering with complete-linkage [11], which is sensitive to noise.

Given real sensor data, there is noise associated with the computation of surface normals. To address this, we employ a Gaussian process (GP) based filter with Thin Plate Spline kernel. This approach fulfills the requirement stated above by integrating both position and normal information into 
similarity measure [57].

Higher sampling frequency for GP centers is used in areas of higher curvature, see Fig. 3 (middle). The distribution of centers captures the geometric similarities (locations and normals) and therefore relate to the similarities in the grasp wrench space [58]. GP partitioning is regulated using a threshold $T_{p}$, so that if $\left|\hat{\phi}_{i, j}\right| \leq T_{p}$, a node is not further divided by GP partitioning but it is split up into all its fingertip units. Nodes consisting of single fingertip units are copied to the next level as long as some other nodes can be partitioned. This guarantees a balanced partitioning tree, and hence a valid surrogate approximation for every level in the hierarchy. As discrete optimization relies on relevant neighbors in the solution space, we introduce connectivity by introducing extra edges between nodes in the same level into $E_{\Phi}$. More precisely, the extended edge set consists of parent-child edges and extra-edges $E_{\Phi}=E_{\Phi}^{P} \cup E_{\Phi}^{E}$ which are given as:

$$
\begin{aligned}
& E_{\Phi}^{P}=\left\{\left\{\phi_{i, j}, \phi_{i-1, k}\right\} \in V_{\Phi} \times V_{\Phi} \mid \hat{\phi}_{i-1, k} \subseteq \hat{\phi}_{i, j}\right\} \\
& E_{\Phi}^{E}=\left\{\left\{\phi_{i, j}, \phi_{i, k}\right\} \in V_{\Phi} \times V_{\Phi} \mid h o p\left(\phi_{i, j}, \phi_{i, k}\right) \leq h\right\}
\end{aligned}
$$

The function $h o p\left(\phi_{i, j}, \phi_{i, k}\right)$ denotes the hop distance between $\phi_{i, j}$ and $\phi_{i, k}$ along edges in $E_{\Phi}^{P}$. The hop limit $h \in \mathbb{N}$ defines the size of the neighborhood and is set to 4 in our experiments, resulting in neighborhoods of size e.g., ca. $4 \mathrm{~cm}$ in the second top level. Using the definitions above, we can now define the $i$-th surrogate approximation of the Fingertip Space $\Phi$ as:

$$
\begin{gathered}
\left(G_{\Phi}\right)_{i}=\left(\left(V_{\Phi}\right)_{i},\left(E_{\Phi}\right)_{i}\right) \\
\left(V_{\Phi}\right)_{i}=\bigcup_{j}\left\{\phi_{i, j}\right\} \\
\left(E_{\Phi}\right)_{i}=\left\{\left\{\phi_{i, j}, \phi_{i, k}\right\} \mid\left\{\phi_{i, j}, \phi_{i, k}\right\} \in E_{\Phi}\right\}
\end{gathered}
$$

which is a subgraph of $G_{\Phi}$ and an approximation at the $i$-th resolution level.

We define the mean location and orientation of the set of fingertip units contained in the partition $\hat{\phi}_{i, j}$ as $\mathbf{p}\left(\hat{\phi}_{i, j}\right) \in \mathbb{R}^{3}$ and $\mathbf{n}\left(\hat{\phi}_{i, j}\right) \in \mathbb{R}^{3}$, this will be used later for stability analysis. In terms of Eq. (2), the penalty assigned to a node $\phi_{i, j}$ is defined as:

$$
w_{i, j}=\frac{1}{\left|\hat{\phi}_{i, j}\right|} \sum_{\phi_{k} \in \hat{\phi}_{i, j}} w_{k}
$$

Given the hierarchy $G_{\Phi}$ of surrogate approximation models, we can optimize a fingertip location in a top-down manner. By optimizing the contact in a coarse to fine fashion, a final contact will be found in the bottom level of the hierarchy. Next, we investigate the grasp synthesis with multiple contacts.

\section{B. Hierarchical Fingertip Space}

In the previous section, we introduced the similarity-based graph $G_{\Phi}$ for a single fingertip. For $m$ fingertips, we define the product graph $\Lambda_{\Phi}=\left(V_{\Lambda}, E_{\Lambda}\right)$ named Hierarchical Fingertip Space (HFTS) as in Eq.(6). Thus, nodes in $V_{\Lambda}$ represent combinations of $m$ contacts, $\lambda_{i, j}=\left(\phi_{i, j_{1}}^{1}, \ldots, \phi_{i, j_{m}}^{m}\right)$, and the graph-distance between nodes in the same level reflects the similarity of the individual contacts. Formally, the HFTS is defined as:

$$
\begin{gathered}
\Lambda_{\Phi}=G_{\Phi}^{1} \times \cdots \times G_{\Phi}^{m} \\
V_{\Lambda}=\left\{\lambda_{i, j}=\left(\phi_{i, j_{1}}^{1}, \ldots, \phi_{i, j_{m}}^{m}\right) \mid \phi_{i, j_{k}}^{k} \in\left(V_{\Phi}^{k}\right)_{i}\right\}
\end{gathered}
$$

where $G_{\Phi}^{k}=\left(V_{\Phi}^{k}, E_{\Phi}^{k}\right), k \in\{1, \ldots, m\}$ is the surrogate hierarchy for the $k$-th fingertip. The penalty value for a set of contacts is defined as minimum of all individual contact penalties:

$$
w_{i, j}^{*}=\min \left\{w_{i, j_{1}}, \ldots, w_{i, j_{m}}\right\}
$$

Optimization on $\Lambda_{\Phi}$ requires definition of neighborhoods and we define two types of edges for $\left.E_{\Lambda}: 1\right)$ Edges between nodes and their parent, $E_{\Lambda}^{P}$, such that $\Lambda_{\Phi}$ inherits the hierarchy levels from the individual $G_{\Phi}^{k}$, and 2) edges between nodes in the same level, $E_{\Lambda}^{E}$, for which the individual contacts are identical or neighbors in their graph $G_{\Phi}^{k}$, respectively. Formally, we obtain $E_{\Lambda}=E_{\Lambda}^{P} \cup E_{\Lambda}^{E}$ :

$$
\begin{aligned}
& E_{\Lambda}^{P}=\left\{\left\{\lambda_{i, j_{1}}, \lambda_{i-1, j_{2}}\right\} \mid \forall k:\left\{\lambda_{i, j_{1}}^{(k)}, \lambda_{i-1, j_{2}}^{(k)}\right\} \in\left(E_{\Phi}^{P}\right)^{k}\right\} \\
& E_{\Lambda}^{E}=\left\{\left\{\lambda_{i, j_{1}}, \lambda_{i, j_{2}}\right\} \mid \forall k:\left\{\lambda_{i, j_{1}}^{(k)}, \lambda_{i, j_{2}}^{(k)}\right\} \in\left(E_{\Phi}^{E}\right)^{k}\right\}
\end{aligned}
$$

where $\lambda_{i, j}^{(k)} \in V_{\Phi}^{k}$ is the $k$-th item of tuple $\lambda_{i, j}$. Similarly to the surrogate models for a single fingertip contact, we define the $i$-th surrogate approximation of multiple fingertip grasping in HFTS as:

$$
\begin{gathered}
\left(\Lambda_{\Phi}\right)_{i}=\left(\left(V_{\Lambda}\right)_{i},\left(E_{\Lambda}\right)_{i}\right) \\
\left(V_{\Lambda}\right)_{i}=\bigcup_{j}\left\{\lambda_{i, j}\right\} \\
\left(E_{\Lambda}\right)_{i}=\left\{\left\{\lambda_{i, j_{1}}, \lambda_{i, j_{2}}\right\} \mid\left\{\lambda_{i, j_{1}}, \lambda_{i, j_{2}}\right\} \in E_{\Lambda}\right\}
\end{gathered}
$$

\section{Grasp Optimization in HFTS}

So far, we described the solution space for grasp synthesis using nodes $\lambda_{g} \in \Lambda_{\Phi}$ from different levels, which are combinations of contacts on the object surface. However, to realize the contacts with a robot hand, we additionally need the joint angles $\mathcal{J}_{g} \in \mathbb{R}^{d}$. A valid grasp solution, $g=\left(\lambda_{g}, \mathcal{J}_{g}\right)$, is a combination of contact positions and joint angles.

1) Grasp Stability: During the pre-grasping phase, when we synthesize a grasp, only visual information of object is available and we need to evaluate or predict grasp stability without feedback. This can be done using contact based force closure analysis [28]: Given a grasp solution $g$, the grasp quality measure $Q\left(\lambda_{g}\right) \in \mathbb{R}$ is the minimum offset between the origin of the wrench space and facets of the convex hull spanned by friction cones of contacts parametrized by positions and normals [23]. The value is positive when the grasp is force closed and larger for more stable grasps.

2) Grasp Reachability: Not all combinations of contacts $\lambda_{g}$ can be realized by a given robotic hand and we can classify contacts into reachable or unreachable using a function $R^{*}: V_{\Lambda} \rightarrow\{0,1\}$ so that the optimization can be constrained to reachable grasps with $R^{*}\left(\lambda_{g}\right)=0$. Since a robotic hand can have many degrees of freedom and complicated coupled kinematics, it can be too costly to analytically compute $R^{*}\left(\lambda_{g}\right)$ in each optimization step. For this, various forms of constraints have been formulated [59], [60]. To achieve required speed and precision, we linearly relax it to a measure of dissimilarity 
between $\lambda_{g}$ and the closest known reachable contacts $\lambda_{g}^{*}$ of grasp solution $g^{*}=\left(\lambda_{g^{*}}, \mathcal{J}_{g^{*}}\right)$. The reachability measure of $\lambda_{g}$ is then reformulated as a residual $R\left(\lambda_{g}\right) \in \mathbb{R}^{+}$:

$$
R\left(\lambda_{g}\right)=\left\|C\left(\lambda_{g}\right)-C\left(\lambda_{g^{*}}\right)\right\|
$$

where $C(\cdot) \in \mathbb{R}^{6 \times(m-2)}$ is an affine invariant encoding of $m$ contacts in terms of its contact locations and normals [61]. Note that a smaller residual indicates more reachable contacts.

To generate a set of viable grasps, we randomly sample hand configurations and save the encoded contacts and corresponding hand configuration $\mathcal{J}_{g}$ into a $k$-d tree like data structure $T$ offline with the query time $\mathcal{O}(n \log n)$. Using $T$, we can compute the residual by lookup and find the hand configuration for realizing the contacts if the residual was small.

$$
T: \lambda_{g} \mapsto\left(\mathcal{J}_{g^{*}}, R\left(\lambda_{g}\right)\right)
$$

3) Grasp Adaptability: We use grasp adaptability to enable finger gaiting already in the grasp synthesis stage. By decomposing the hand Jacobian and calculating the manipulability [62] of a hand configuration in the tangential plane of contacts, we measure the adaptability of a grasp, denoted as $A\left(\mathcal{J}_{g}\right) \in$ $\mathbb{R}^{+}$. Concretely, given the Jacobian $J_{f}\left(\mathcal{J}_{g}\right) \in \mathbb{R}^{3 \times n}$ and the normal $\mathbf{n}_{f} \in \mathbb{R}^{3}$ of fingertip $f$, the Jacobian can be rotated by $R_{f} \in \mathbb{R}^{3 \times 3}$ such that the last row of $\hat{J}_{f}\left(\mathcal{J}_{g}\right)=R_{f} J_{f}\left(\mathcal{J}_{g}\right)$ corresponds to the movement of fingertip in the direction of $\mathbf{n}_{f}$. The first two rows of $\hat{J}_{f}$, denoted by $\widetilde{J_{f}}\left(\mathcal{J}_{g}\right) \in \mathbb{R}^{2 \times n}$, are then the projection of $J_{f}$ in the tangential plane of the fingertip normal.

$$
A\left(\mathcal{J}_{g}\right)=\sum_{f} \sqrt{\operatorname{det}\left(\widetilde{J_{f}}\left(\mathcal{J}_{g}\right){\widetilde{J_{f}}}^{T}\left(\mathcal{J}_{g}\right)\right)}
$$

Note that we can assume that the fingertip normal (on the robot hand) and the fingertip unit normal will be similar when the grasp is realized if $R\left(\lambda_{g}\right)$ is small. An example of grasp adaptability measure is shown in Fig. 5 Since this measure is hand configuration based, it is affine invariant, and hence grasp pose independent.

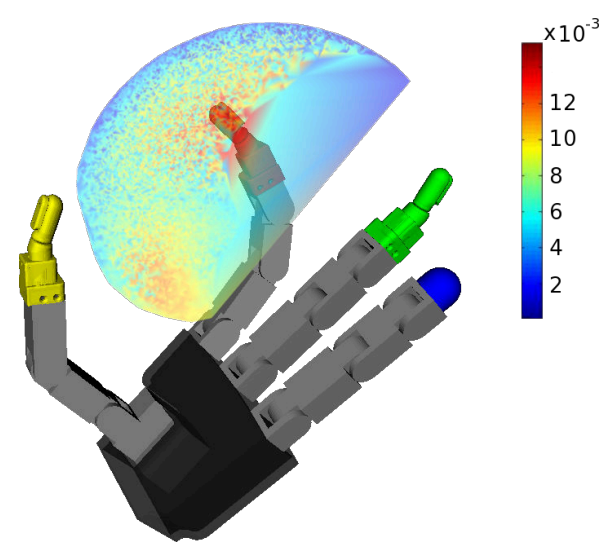

Fig. 5. Grasp Adaptability for fingertip 1(red). Adaptability is computed for fingertip positions sampled in joint space. The colored volume shows finger adaptability values at sampled fingertip positions.

In order to capture grasp stability, reachability and adaptability in the grasp optimization, the optimization objective is defined as follows:

$$
\begin{array}{ll}
\text { Priority 1: Maximize } & \theta(g) \\
\text { Priority 2: Maximize } & A\left(\mathcal{J}_{g}\right)
\end{array}
$$

with

$$
\theta(g)=Q\left(\lambda_{g}\right)-\alpha R\left(\lambda_{g}\right), \quad \alpha \in \mathbb{R}^{+}
$$

where $\alpha$ is a weighing factor to account for the hand size, which is determined by the range in which the grasp quality values $Q\left(\lambda_{g}\right)$ vary, as it is related to the grasp sizes [28]. To optimize the second objective, we use a sorted lookup table for $R\left(\lambda_{g}\right)$ which returns the most adaptable joint configuration in the area of the best grasp according to $A\left(\lambda_{g}\right)$ [63], [64] when querying reachability residuals (line 7 and 10 in Alg. 11). As we can see in Fig. 6, for the same contact locations, there can be multiple hand configurations for realizing it, however, our prioritized lookup table will always return the hand configuration with the best adaptability.
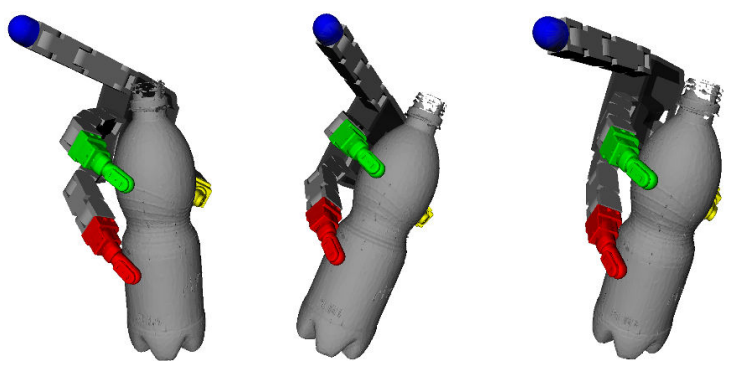

Fig. 6. Grasps with same contacts and different adaptabilities: the left grasp has the highest adaptability.

Having defined the objective function, we can now proceed to grasp synthesis. Using a surrogate-based optimization metaheuristic, we need to find solutions on each of the surrogate approximations and extend them to the next model. For optimization in each model, we adopt stochastic hill climbing which can escape from local optima by means of randomness. Switching from solution $g$ to $g^{\prime}$ is determined by the probabilistic function in Eq. (16):

$$
\operatorname{Pr}\left(g, g^{\prime}\right)=\left(1+\exp \frac{w_{g} \theta(g)-w_{g^{\prime}} \theta\left(g^{\prime}\right)}{\zeta}\right)^{-1}
$$

where $w_{g}$ is the penalty assigned to a tuple of contacts defined by Eq. (7). The randomness in the optimization is determined by $\zeta$, it makes the optimization more random when a large value is chosen, while it behaves more like pure hill climbing if a small value is applied. The grasp optimization algorithm is shown in Alg. 1.

For realizing the grasp, we can transform the hand base to the pose where the fingertips meet the contact locations [11]. In cases when the final reachability residual $R\left(\lambda_{g}\right) \neq 0$, a local optimization of joint configuration by linear interpolation [65] is required to realize desired contacts. To avoid too small and time consuming incremental improvements at each level, we utilize a stopCondition. It can be set to false if we want to explore the space until convergence or we control the number of iterations by setting a threshold for the optimization function in Eq. (15). 


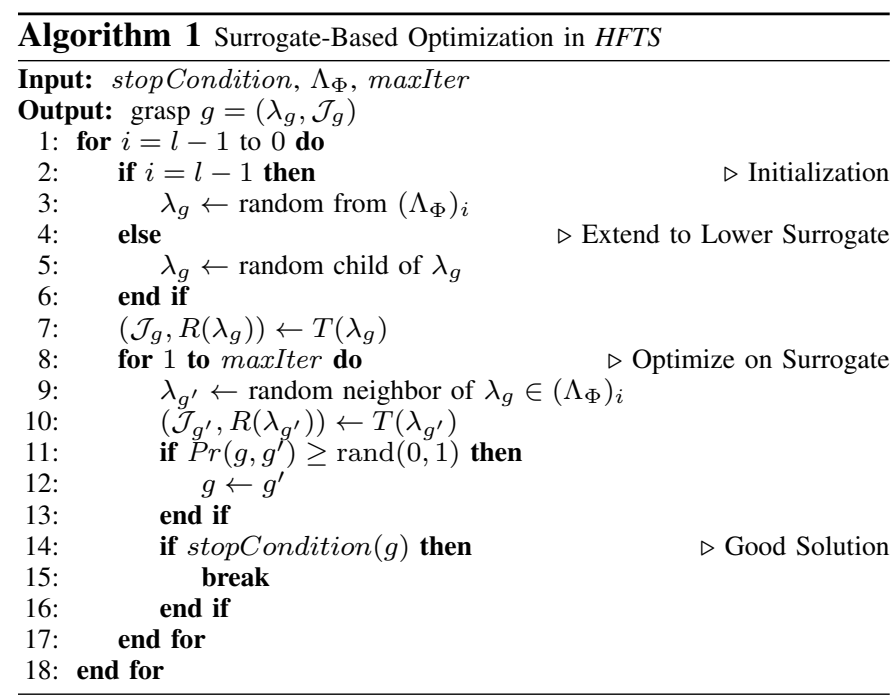

\section{GRASP ADAPTATION}

A synthesized grasp is executed using a simple position control. When contacts are made and tactile readings are available, an object-level impedance controller [3] is used to regulate grasp forces. The object-level impedance control for dexterous robotic hands is still an open question and is currently feasible for 3 fingers or 4 fingers with virtual linkage [66]. For the demonstration of the entire system, although we have shown that we are able to plan grasps for $m$ fingertips, we will in the rest of the paper explain the control and adaptation of grasps by examples of only 3 fingers.

The grasp impedance controller is formulated in a virtual frame (VF) defined in terms of fingertip locations as

$$
\begin{aligned}
R_{o} & =\left[\mathbf{v}_{x}, \mathbf{v}_{y}, \mathbf{v}_{z}\right] \in \mathrm{SO}(3) \\
\mathbf{v}_{x} & =\frac{\mathbf{p}_{3}-\mathbf{p}_{1}}{\left\|\mathbf{p}_{3}-\mathbf{p}_{1}\right\|} \\
\mathbf{v}_{z} & =\frac{\left(\mathbf{p}_{2}-\mathbf{p}_{1}\right) \times \mathbf{v}_{x}}{\left\|\left(\mathbf{p}_{2}-\mathbf{p}_{1}\right) \times \mathbf{v}_{x}\right\|} \\
\mathbf{v}_{y} & =\mathbf{v}_{z} \times \mathbf{v}_{x}
\end{aligned}
$$

where $\mathbf{p}_{1}, \mathbf{p}_{2}$ and $\mathbf{p}_{3} \in \mathbb{R}^{3}$ are locations of the fingertips, see Fig. 7
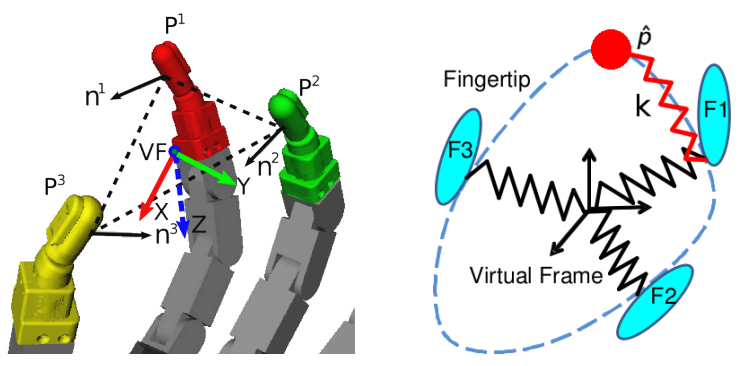

Fig. 7. Left: Virtual frame $R_{o}, v_{x}, v_{y}$ and $v_{z}$ defined by fingertip locations. Right: Virtual springs used by the impedance controller. A virtual spring (red) is superimposed on the impedance controller (between fingertip and the new location $\hat{p}$ ) when a fingertip gaiting is requested.

A grasp in the $\mathrm{VF}$ is denoted $\hat{g}=(K, L, S)$ where $K=\left(K_{x}, K_{y}, K_{z}\right) \in \mathbb{R}^{3}$ is the grasp stiffness and $L=$
$\left(L_{1}, L_{2}, L_{3}\right) \in \mathbb{R}^{3}$ is the grasp rest length, i.e. the distance between each fingertip and the center of VF. $S=\left(S_{1}, S_{2}, S_{3}\right) \in$ $\mathbb{R}^{57}$ denotes the tactile readings, in our case from SynTouch sensors.

Grasp stability is monitored using a probabilistic representation relying on a Gaussian Mixture Model $\Theta$ that is trained offline, see Fig. 8. As described in detail in our previous work [9], $\Theta$ is trained over $K, L, S$ parameters for a variety of objects. Given $\Theta$, grasp stability is estimated by

$$
p(\hat{g} \mid \Theta)=\sum_{i=1}^{n_{g}} \pi_{i} \mathcal{N}\left(\hat{g} \mid \mu_{i}, \Sigma_{i}\right)
$$

where $n_{g}$ is the number of Gaussian components, each of which has a prior $\pi_{i} \cdot \mathcal{N}\left(\hat{g} \mid \mu_{i}, \Sigma_{i}\right)$ is the Gaussian distribution with mean $\mu_{i}$ and covariance $\Sigma_{i}$.

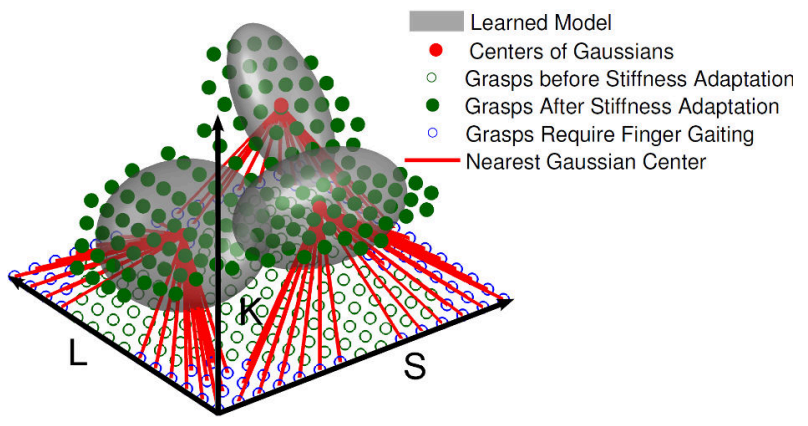

Fig. 8. GMM model $\Theta$ for grasp stability estimation and decision making for grasp adaptation. The gray ellipsoids depict the Gaussian components of $\Theta$, dots and circles show grasps, represented by $K, L, S$ in different stages, and the red lines illustrate how grasps are switched between different stages by grasp stiffness adaptation and fingertip gaiting.

A grasp $\hat{g}$ is considered unstable if the log likelihood of Eq. (18) is smaller than a predefined threshold determined by the ROC curve [9]. If a grasp $\hat{g}$ is unstable, we compute its Mahalanobis distance to each component in $\Theta$ and denote the minimum distance as $m_{d}$. If $m_{d}$ is within two standard deviations, we apply force adaptation by changing stiffness $K$ to the value obtained by computing the maximum expectation of $K$ conditioned on $L$ and $S$. The details of this process have been described in detail in our previous work [9]. Otherwise, a finger gaiting strategy is initiated as explained in detail in next section.

\section{REGRASPING BY FINGER GAITING}

Stiffness adaptation is not enough in cases when there is an upper bound on the force can be exerted by the hand. Thus, to stabilize a grasp, the system initiates finger gaiting. Finger gaiting is defined as an optimization problem based on the current rest length $L$ represented in VF:

$$
\theta^{*}\left(\lambda_{g}\right)=\left\|L-L^{*}\right\|+\beta R\left(\lambda_{g}\right)
$$

where $R\left(\lambda_{g}\right)$ is the reachability defined in Eq. [10], $\beta \in \mathbb{R}^{+}$ is a weighing factor to account for the hand size, as $L$ values range differently in terms of hand sizes. $L^{*}$ is the desired rest length obtained from the closest Gaussian center $\hat{g}^{*}=$ $\left(K^{*}, L^{*}, S^{*}\right)$ in terms of $m_{d}$. The reasoning above is to find 


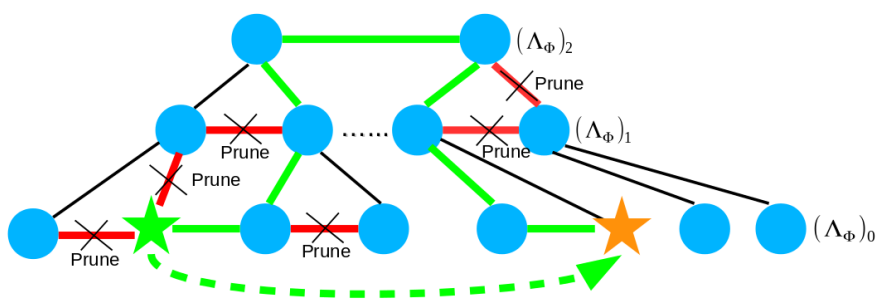

Fig. 9. Breadth-first search in HFTS for fingertip gaiting optimization. The green path shows how the search fringe evolves, and the red edges show the pruned path due to the 2 criteria defined in Alg. 2

the closest stable and reachable grasp to the current one, taking into account the current tactile readings.

For the robot hand we use in this work, we can only relocate fingertip $F 1$ or $F 2$, as shown in Fig. 7 since relocating the thumb $F 3$ leaves the grasp without contacts on the opposite side of the object. Our strategy of choosing between $F 1$ and $F 2$ is straightforward: we compute the optimization for $F 1$ and $F 2$ in parallel for minimizing the objective value from Eq. (19), the one resulted with smaller values is chosen. Our optimization procedure employs breadth-first search in $\Lambda_{\Phi}$ starting from the initial contact. The search is terminated in a branch if the reachability measure grows beyond a predefined threshold $\epsilon_{R}$. Since we move only one finger, we need an additional rule:

$$
\text { Prune }\left(\lambda_{g}, \lambda_{g^{\prime}}, f_{o}\right)= \begin{cases}\text { True }, & \exists i: i \neq f_{o} \wedge \lambda_{g}^{(i)} \neq \lambda_{g^{\prime}}^{(i)} \\ \text { False, } & \text { otherwise. }\end{cases}
$$

where $f_{o}$ is the fingertip to be relocated, $\lambda_{g}$ is the node that represents the current grasp contacts, and $\lambda_{g^{\prime}}$ is the new solution. Since the search fringe can go upwards in the hierarchy graph $\Lambda_{\Phi}$, this rule asserts that only a single fingertip is moved while the remaining two are fixed. The main idea is sketched in Fig. 9 and the procedure summarized in Alg. 2. Note that it includes the penalty factor from Eq. (7).

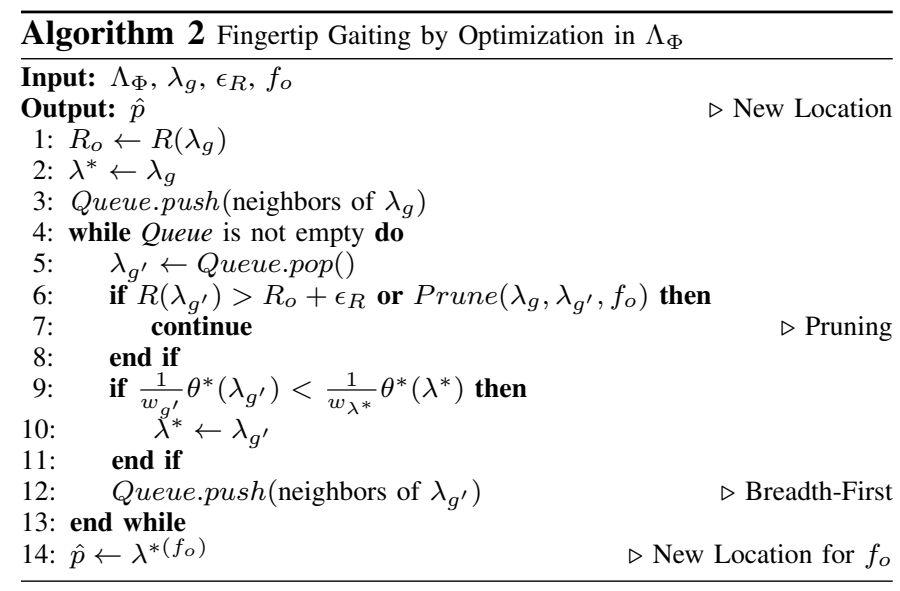

\section{A. Fingertip Gaiting in Practice}

When grasp stability changes rapidly and finger gaiting is triggered frequently, to avoid switching between impedance and position control, we stay in impedance control mode during finger gaiting by sliding the finger to the desired position. To allow this, we formulate fingertip gaiting using impedance controller defined in VF. A virtual spring with stiffness $k$ is defined to connect the current location of the moved fingertip and $\hat{p}$, which is equivalent to a fingertip impedance controller superimposed on the original grasp controller. An example of fingertip $F 1$ gaiting is depicted as in Fig. 7 .

The stiffness $k$ of the virtual spring is determined by the distance $d_{\hat{p}} \in \mathbb{R}$ between the fingertip's current location and $\hat{p}$, and an empirical parameter $\Gamma \in \mathbb{R}$ as: $k=d_{\hat{p}} \Gamma$. In this way, the fingertip will be slided towards $\hat{p}$ while keeping the contact on the object. Since $\hat{p}$ is computed in the HFTS, we ensure that the desired position is on the object surface. If a new goal position is requested during finger gaiting, the system will either continue to the new position if the same fingertip is concerned, or stop the current gaiting and initiate gaiting with another fingertip. An example situation is depicted in Fig. 10 where fingertip $F 2$ stopped moving before the desired position is reached, since the grasp was estimated as stable on the way.
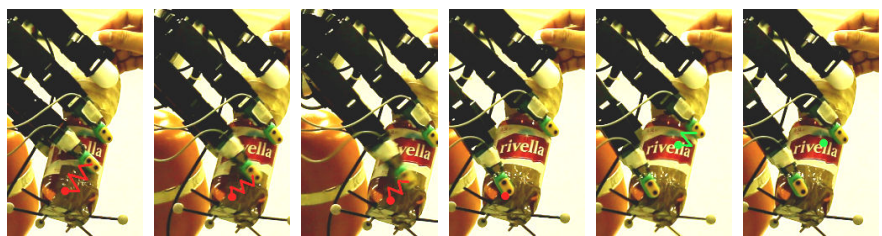

Fig. 10. The rivella bottle is grasped by the Allegro hand and a human is applying random perturbations on top of it. The red and green points are showing the new locations for fingertip $F 1$ and $F 2$ computed by Alg. 2 with virtual springs in the virtual frame.

\section{EXPERIMENTAL EVALUATION}

We perform experimental evaluations with an Allegro hand mounted on a Kuka LWR arm. The hand is equipped with SynTouch tactile sensors on three fingertips. The systems performance is evaluated using six objects shown in Fig. 11 . which are tracked using the OptiTrack ${ }^{2}$ real-time motion tracking system. The evaluations presented below demonstrate the performance of the grasp synthesis system alone as well as the integrated system for grasp adaptation.

\section{A. Grasp Synthesis}

Grasp synthesis is performed on a point cloud representation of objects obtained offline. We also generated a reachability table with $10^{6}$ hand configurations using rejection sampling: configurations are first uniformly sampled in the hand joint space and we keep those collision-free configurations with adaptabilities larger than 0.02 , which is determined empirically since we observed that the grasps are rarely adaptable with adaptabilities lower than 0.02 .

Alg. 1 generates both contact locations and hand configurations. Simple position based control is used to execute a grasp [9]. A few examples are shown in Fig. 11 and Fig. 12 .

\footnotetext{
${ }^{2}$ http://www.naturalpoint.com/optitrack/
} 

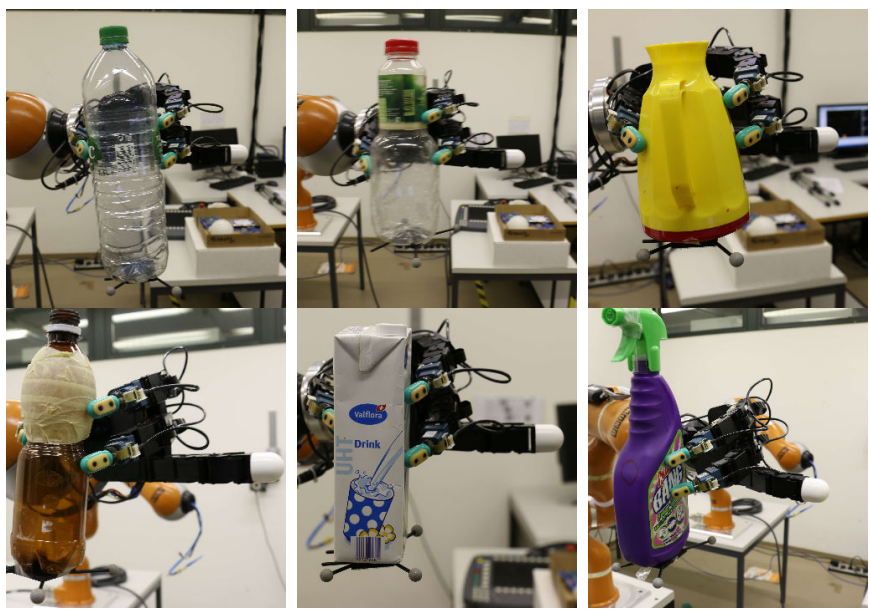

Fig. 11. Six example objects used in the evaluation: there is both variation in global geometry as well as local surface properties. From top-left to bottomright: bottle1, bottle2, jug, rivella, milk and spray.
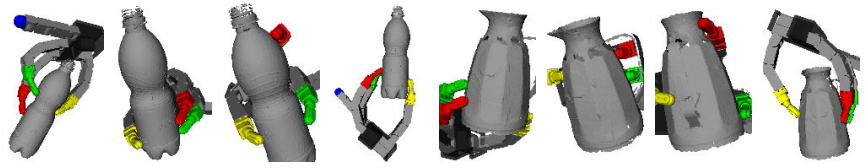

Fig. 12. Example grasps generated by Alg. 1 with stopCondition $(g)$ that, as soon as the grasp is stable and the reachability residual is smaller than 0.006 , we stop optimizing on the current level of $\Lambda_{\Phi}$ and continue on the next level.

For evaluating the performance of the grasp planner, we repeat the grasp optimization according to Alg. 1 for each test object. In order to keep an equal number of iterations for each repetition of the algorithm, we set maxIter $=100$ and $\operatorname{stopCondition}(g)=$ false. For each object, we run the algorithm with random initialization until we achieve 100 stable and collision free grasps. Evaluation results are summarized into a table shown in Fig. 13 .

\begin{tabular}{|c|c|ccccc|}
\hline Object(\#Units) & $T_{p}$ & \#Levels & \#Nodes & Time(s) & SR(\%) & $\theta\left(\times 10^{-2}\right)$ \\
\hline bottle1(2736) & & 6 & 5672 & 1.81 & 96.15 & 5.23 \\
bottle2(3102) & & 6 & 6321 & 1.77 & 93.46 & 4.74 \\
jug(2671) & \multirow{2}{*}{10} & 5 & 3227 & 1.64 & 89.29 & 4.96 \\
rivella(2273) & & 6 & 5124 & 1.54 & 98.03 & 3.29 \\
milk(2696) & & 5 & 3204 & 0.96 & 97.09 & 4.05 \\
spray(3207) & & 6 & 6926 & 2.04 & 94.34 & 3.71 \\
\hline bottle1(2736) & & 3 & 2839 & 0.45 & 94.34 & 5.01 \\
bottle2(3102) & & 3 & 3203 & 0.64 & 94.34 & 4.56 \\
jug(2671) & \multirow{4}{*}{40} & 4 & 3002 & 0.81 & 87.72 & 4.99 \\
rivella(2273) & & 3 & 2399 & 0.44 & 99.01 & 3.17 \\
milk(2696) & & 4 & 2912 & 0.73 & 96.15 & 4.12 \\
spray(3207) & & 3 & 3310 & 0.62 & 92.59 & 3.62 \\
\hline
\end{tabular}

Fig. 13. Evaluation of Alg. 1 \#Units: number of fingertip units in $\Phi(\mathcal{P})$, \#Levels: number of levels in graph $G_{\Phi}$ (including the top level with only one node), \#Nodes: number of nodes in graph $G_{\Phi}$, Time(s): average time in seconds for one run of the algorithm, SR: success rate of synthesizing a stable grasp. $\theta$ : optimized objective value defined in Eq. (15). The evaluations were implemented in Python on a machine with Ubuntu 12.04 running on Intel Core i7-2820QM 2.30GHz processors.

First, Fig. 13 shows that the number of levels of the graph $G_{\Phi}$ are between 4 and 5 when $T_{p}=10$, or between 3 and 4 when $T_{p}=40$. This indicates that our system produces similar depth of the HFTS independent of the shape of the object. However, the shape of the object affects the number of nodes at each level, given that some branches are terminated earlier for objects of simpler geometry, such as the milk package. It is worthwhile to note that, the partitioning of rivella ended up with more levels than milk, and this is reverted when it was $T_{p}=40$. This is due to the fact that if one sets a small threshold $T_{p}$, a larger sub-partition would continue being partitioned, where as smaller ones are terminated earlier. This causes the rivella to have more levels when $T_{p}$ is smaller, due to its uneven sub-partitioning in the lower levels of the hierarchy.

Regarding the success rate (SR), we can see that SR lies at approximately $90 \%$. Fig. 14 shows average adaptabilities for the 100 stable grasps for each object. Average adaptability values, computed by Eq. (12), are large showing that our methodology considers the adaptability effectively.

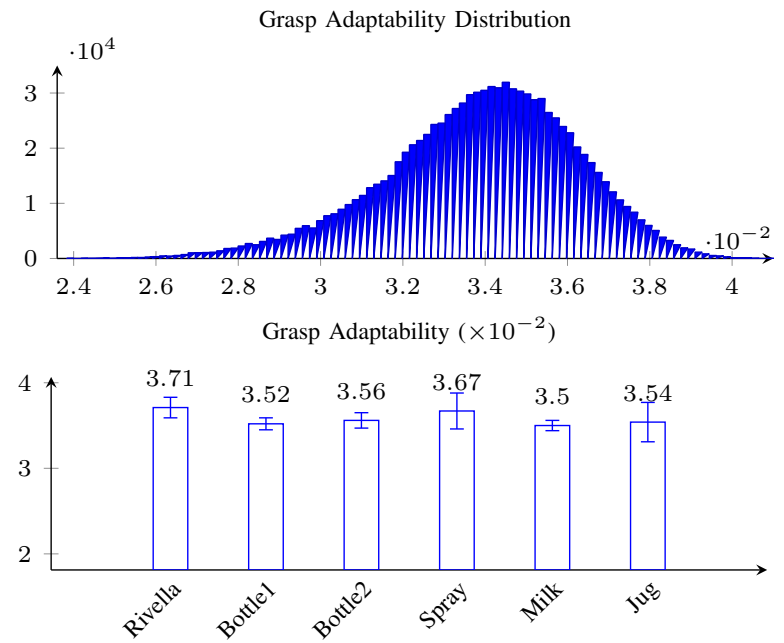

Fig. 14. Upper: Grasp adaptability distribution of $10^{6}$ hand configurations in the reachability lookup table. Lower: Average grasp adaptabilities for the 100 grasps generated in the evaluations for all objects.

\section{B. Grasp Adaptation}

Once a grasp is executed and contacts established, the system will enter the post-grasping phase and start monitoring the stability based on tactile feedback. Instead of position control, the impedance controller is used to control the grasp using GMM based model $\Theta$. The log likelihood threshold for Eq. (18) is set to -100 in terms of the ROC curve with a false positive rate $F P R=15 \%$ [9]. For the force control of the hand, we set the initial grasp stiffness $K=(12,2,2)$ and use it for the execution of all grasps, as described in Sec. IV

For the evaluation, we run two sets of experiments: 1) We continuously increase the objects' weight by filling them to evaluate the maximum weight each grasp can withstand, and 2) we shake the grasped objects by linearly increasing acceleration in different directions to evaluate the maximum acceleration each grasp can withstand. For comparison, we conduct the same experiments without any grasp adaptation and on the system proposed in [9] which does not consider object shape information when relocating fingertips.

1) Testing maximum weight: For each object, we execute the best out of 100 grasps generated in Sec. VI-A and align the object with vertical axis as shown in Fig. 18 We then 
gradually fill object with black pepper beans and record the maximum weight the grasp can withstand. The maximum weight is reached when the stability estimator predicts unstable grasp for more than 2 seconds or if the object drops. We repeat this test for each grasp 5 times and summarize the results in Fig. 15

\begin{tabular}{|c|c|c|c|c|}
\hline Object & Weight & Without & With $[9]$ & Improved \\
\hline bottle1 & 34 & $55.1 \pm 7.11$ & $153.1 \pm \overline{12.31}$ & $165.3 \pm 13.27$ \\
\hline bottle2 & 39 & $62.8 \pm 6.63$ & $102.3 \pm 13.38$ & $121.3 \pm 9.91$ \\
\hline jug & 112 & $125.3 \pm 14.90$ & $147.4 \pm 9.62$ & $162.1 \pm 13.12$ \\
\hline rivella & 24 & $36.0 \pm 6.96$ & $76.5 \pm 9.4$ & $92.7 \pm 7.45$ \\
\hline milk & 34 & $63.5 \pm 8.20$ & $151.8 \pm 7.24$ & $157.4 \pm 8.35$ \\
\hline spray & 63 & $75.7 \pm 7.21$ & $102.2 \pm 6.02$ & $121.6 \pm 7.15$ \\
\hline
\end{tabular}

Fig. 15. The comparison of the supported object weights $($ mean \pm std, Unit:gram). without: without grasp adaptation; with [9]|: with grasp adaptation in [9]; improved: the adaptation approach proposed in this paper.

Naturally, the system without any adaptation performs the worst and the integrated system outperforms the system from [9]. This is since our system: i) takes into account grasp reachability during the exploration, and ii) the new location is computed in the HFTS, thus ensuring it is valid, avoiding problems shown in Fig. 16, and iii) considers two fingers for gaiting, resulting in increased flexibility.

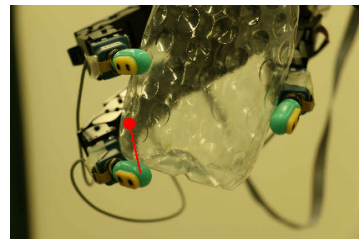

Fig. 16. The risk of moving a fingertip to an non-existing position present in [9] is addressed by using our HFTS representation. The red point shows the fingertip position before gaiting.

A quantitative evaluation of the proposed system and the system in [9] has been conducted with respect to optimization residual. We first execute the grasp in simulation and then trigger the fingertip gaiting by sending desired rest lengths randomly sampled around the current values within a ball of radius $20 \mathrm{~mm}$. The result is shown in Fig. 17. Due to the object shape constraint, the systems cannot provide zero residuals. Our system performs much better for non-planar objects given that HFTS representation considers shape in an effective way.

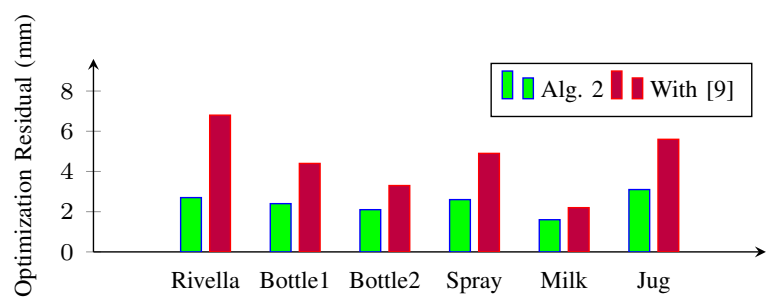

Fig. 17. The results of fingertip gaiting optimized residual $\left\|L-L^{*}\right\|$ from Eq. 19. $10^{5}$ desired rest lengths are randomly sampled around the current rest length within a ball of radius $20 \mathrm{~mm}$.

An example of the supported weight test for the rivella bottle is shown in Fig. 18 . In the beginning when the object is not too heavy, the likelihood $p(\hat{g} \mid \Theta)$ is larger than -100 and the grasp stiffness $K$ is constant. As the weight increases, the grasp becomes unstable and stiffness adaptation is initiated. Stiffness changes rapidly when the weight increases, and when the force adaptation is not able to handle the current weight, a finger gaiting is triggered and fingertip $F 2$ is relocated. After finger gaiting, grasp stiffness is decreased since the new grasp requires less force to be stable. As the weight increases again, the whole process is repeated, resulting in $F 1$ finger gaiting.
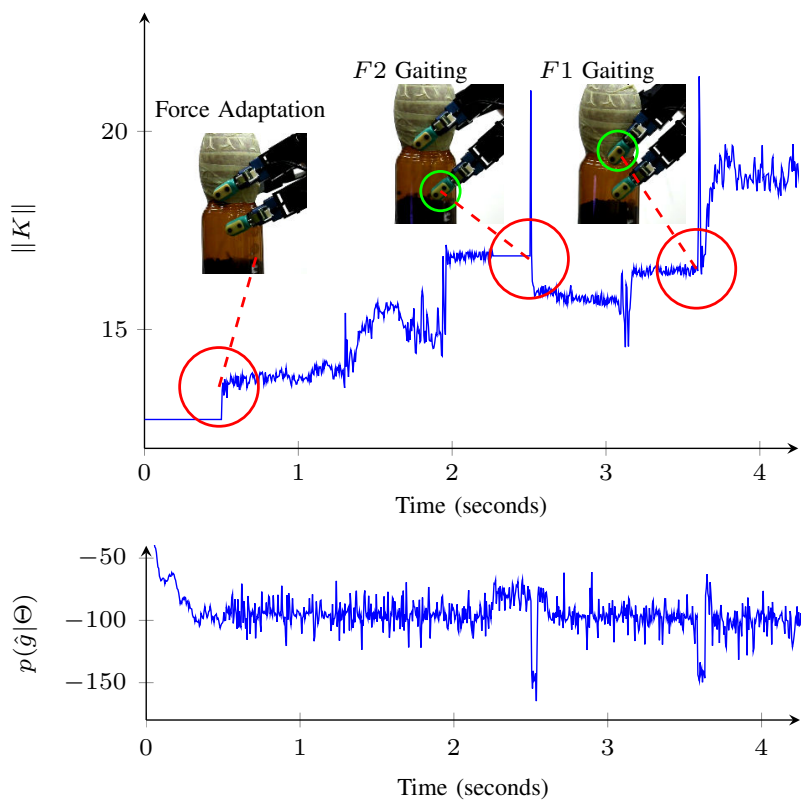

Fig. 18. A record of supported weight test of a grasp on rivella bottle. Upper: The norm of grasp stiffness and fingertip gaiting. Lower: Likelihood for grasp stability estimation using $\Theta$ defined in Eq. 18 .

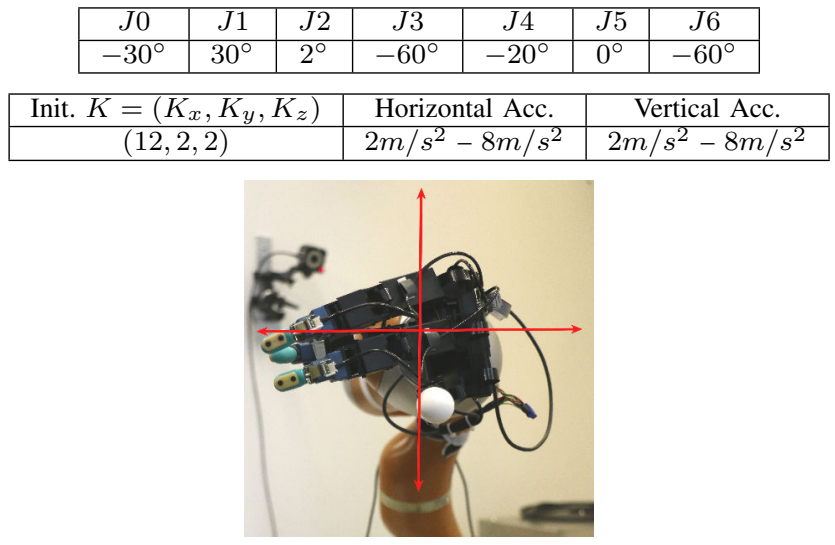

Fig. 19. The setup of grasp shaking test, in which the arm shakes each grasp in horizontal and vertical directions. $J 0$ to $J 6$ are the joint values for the initial pose of shaking test. When shaking horizontally, the shaking direction is fixed to be perpendicular to the palm.

2) Shaking Test: External disturbances, such as collisions, may occur once a grasp has been executed. To evaluate the proposed system, we designed a shaking test. We first execute the best out of the 100 generated grasps for each object according to Eq. (15), and then pose the arm to the configuration shown in Fig. 19. Thereafter, we start to shake the arm in either vertical or horizontal directions while linearly increasing the 

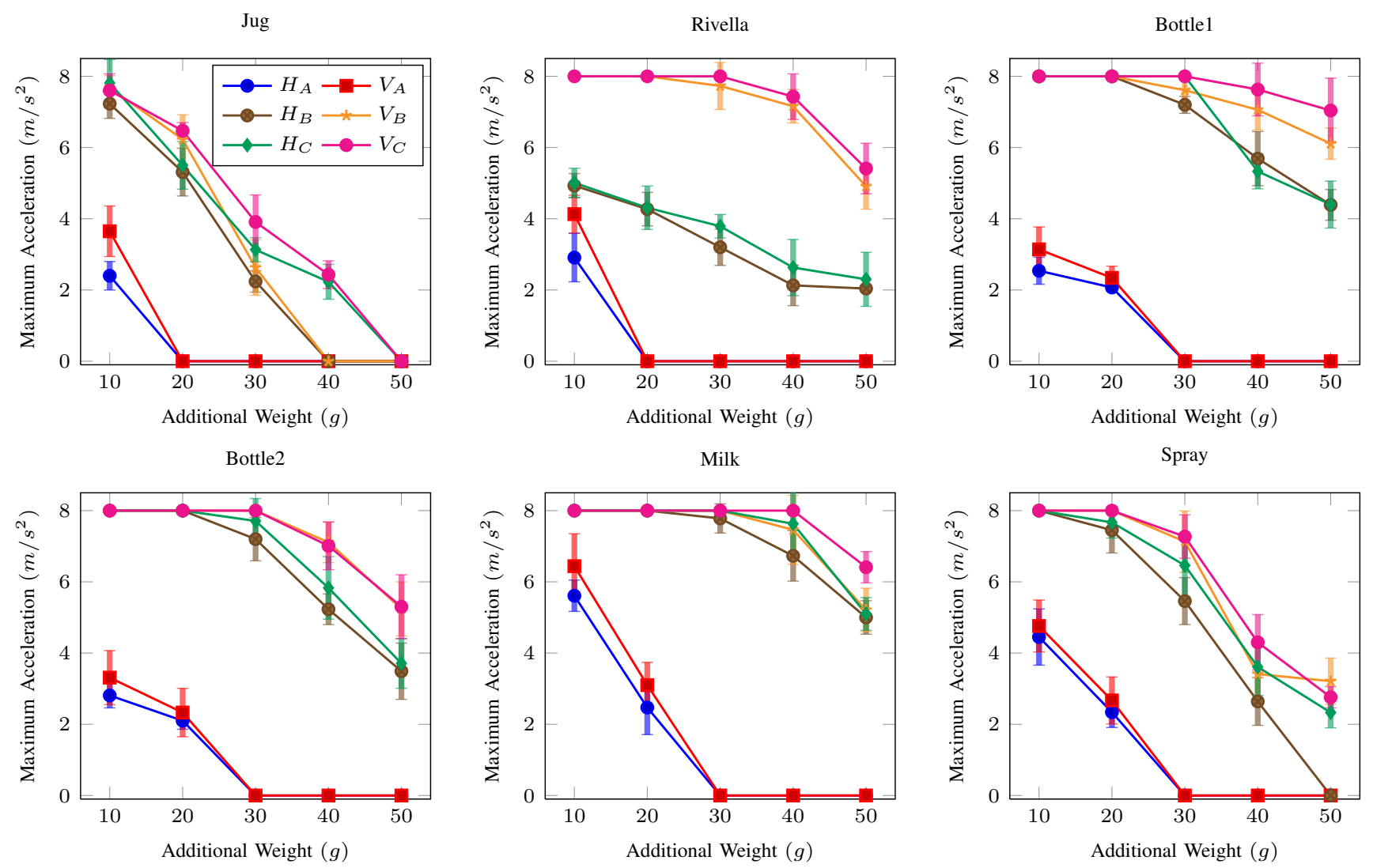

Fig. 20. Results of shaking tests. In the legend, $H$ and $V$ refer to horizontal shaking test and vertical shaking test respectively. $A, B$ and $C$ refer to 3 grasp strategies: grasp without adaptation, grasp adaptation in $[9]$ and the grasp adaptation proposed in this paper. The larger the maximum acceleration rate shown in the graph is, the more external disturbances a grasp could withstand during the tests.

\begin{tabular}{c|cccccc}
\hline Object & Avg. Duration(ms) & Avg. Improvement & Avg. Comp. Time(ms) & Avg. Err.(m) & Avg. \# Nodes & \# Gaiting \\
\hline bottle1 & 261.2 & 66.21 & 30.7 & 0.0074 & 279.2 & 6 \\
bottle2 & 320.1 & 75.17 & 32.1 & 0.0062 & 221.5 & 4 \\
jug & 414.4 & 70.72 & 19.4 & 0.0042 & 140.4 & 5 \\
rivella & 447.9 & 52.39 & 38.6 & 0.0045 & 194.1 & 12 \\
milk & 392.6 & 47.11 & 24.9 & 0.0057 & 137.6 & 9 \\
spray & 502.7 & 57.26 & 29.2 & 0.0068 & 197.6 & 7 \\
\hline
\end{tabular}

Fig. 21. Results for the horizontal shaking tests when the objects are filled with $20 \mathrm{~g}$ of pepper beans (from left to right): average duration for one time of fingertip gaiting; Average stability likelihood improvement after fingertip gaiting; Average computation time of Alg. 2 for each computation; Average errors between achieved rest lengths and the rest lengths computed by Alg. 2 Average number of nodes explored in Alg. 2. Number of fingertip gaiting required during a shaking test with 14 shakes. The evaluations were implemented in C++ and run on a machine with Ubuntu 12.04 running on Intel Core i7-2820QM $2.30 \mathrm{GHz}$ processors.

acceleration from $2 \mathrm{~m} / \mathrm{s}^{2}$ to $8 \mathrm{~m} / \mathrm{s}^{2}$. The shaking magnitude is limited to $10 \mathrm{~cm}$ in either directions, which means that the hand is accelerating in the first $5 \mathrm{~cm}$ and decelerating in the second $5 \mathrm{~cm}$. After every period of shaking, we increase the acceleration by $1 \mathrm{~m} / \mathrm{s}^{2}$ and therefore have 14 shakes for every test.

Similarly to the supported weight test, we evaluate each grasp by measuring the maximum acceleration it can withstand. The criterion is similar: the maximum acceleration is recorded when the grasp is predicted as unstable for more than 2 seconds or if the object drops. The shaking test is conducted in both directions separately and on each object by filling it with $10 g, 20 g, 30 g, 40 g$ and $50 g$ black pepper beans. Each test is repeated 5 times.

Experimental results are summarized in Fig. 20. If the maximum acceleration rate is $8 \mathrm{~m} / \mathrm{s}^{2}$, it means that the grasp has been kept stable during the test. On the other hand, if the maximum acceleration rate is $0 \mathrm{~m} / \mathrm{s}^{2}$, it means that the grasp could not withstand any shaking. We can see that our system outperforms both the system without adaptation and the system proposed in [9]. The advantage of our approach is that we ensure that the finger gaiting has resulted in an actual contact with the object which is not the case in [9]. In addition, the flexibility of gaiting two fingers provides additional strength.

Additional quantitative results are shown in Fig. 21. We can see that the average computing time of Alg. 2 is between $20 \mathrm{~ms}$ and $40 \mathrm{~ms}$. The average number of explored nodes shows that the pruning is efficient since less than $5 \%$ of all nodes in $G_{\Phi}$ are considered. Note that the computation time and number of explored nodes are heavily dependent on the connectivity of graph $G_{\Phi}$ : less nodes in the graph does not mean less computing time. Therefore, the connectivity in $G_{\Phi}$ indirectly 
measures how complex an object is in the context of this system.

\section{CONCLUSION}

We have presented a unified framework for grasp planning and in-hand grasp adaptation using visual, tactile and proprioceptive feedback. The proposed Hierarchical Fingertip Space defines a hierarchy of surrogate solution spaces of fingertip grasping enabling both planning and adaptation. By augmenting the fingertip space in terms of local geometry and spatial relations, as well as optimizing hand configurations with respect to grasp adaptability, we demonstrated efficient planning and adaptation. Moreover, the probabilistic model for grasp stability estimation and adaptation has shown its feasibility in closing the loop between grasp replanning and control. We have evaluated the performance of the proposed system quantitatively and shown that the proposed system significantly improves the robustness of grasp execution. It also outperforms our previous work reported in [9]. To the best of our knowledge, this is so far the first system that accomplishes grasp synthesis, stability estimation, online replanning and inhand adaptation in a unified framework, as well as evaluating this on a real physical system.

However, as a basic drawback of most learning based approaches, our probabilistic model is experience based, and hence relying on the training data, i.e. limited number of objects and examples to generalize from. As a potential future work, we plan to design an active learning strategy to update this model iteratively using new experiences over time, so as to evolve the model in a long term to generalize it to a broader set of objects, without retraining the models from scratch. Additionally, in case of more complicated object shapes, to move the fingertip compliantly on the object surface requires a more sophisticated controller and planning method, which we would like to look into as our future work.

\section{ACKNOWLEDGEMENT}

This work was supported by the European Union Seventh Framework Program FLEXBOT - FP7-ERC-279933 and ROBOHOW - FP7-288533.

\section{REFERENCES}

[1] K. Shimoga, "Robot grasp synthesis algorithms: A survey," The International Journal of Robotics Research, vol. 15, no. 3, pp. 230-266, 1996.

[2] C. Borst, M. Fischer, and G. Hirzinger, "Calculating hand configurations for precision and pinch grasps," in Proc. IEEE/RSJ Int. Conf. Intelligent Robots and Systems, 2002.

[3] K. Tahara, S. Arimoto, and M. Yoshida, "Dynamic object manipulation using a virtual frame by a triple soft-fingered robotic hand," in Proc. IEEE Int. Conf. Robotics and Automation, 2010.

[4] T. Yoshikawa, "Multifingered robot hands: Control for grasping and manipulation," Annual Reviews in Control, vol. 34, no. 2, pp. 199 208, 2010.

[5] J. Romano, K. Hsiao, G. Niemeyer, S. Chitta, and K. Kuchenbecker, "Human-inspired robotic grasp control with tactile sensing," IEEE Transactions on Robotics, vol. 27, no. 6, pp. 1067-1079, Dec 2011.

[6] A. Sahbani, S. El-Khoury, and P. Bidaud, "An Overview of 3D Object Grasp Synthesis Algorithms," Robotics and Autonomous Systems, vol. 60, no. 3, pp. 326-336, 2011.

[7] Y. Bekiroglu, D. Song, L. Wang, and D. Kragic, "A probabilistic framework for task-oriented grasp stability assessment," in Proc. IEEE Int. Conf. Robotics and Automation, 2013.
[8] K. Hang, J. A. Stork, F. T. Pokorny, and D. Kragic, "Combinatorial optimization for hierarchical contact-level grasping," in Proc. IEEE Int. Conf. Robotics and Automation, 2014.

[9] M. Li, Y. Bekiroglu, D. Kragic, and A. Billard, "Learning of grasp adaptation through experience and tactile sensing," in Proc. IEEE/RSJ Int. Conf. Intelligent Robots and Systems, 2014.

[10] J. Bohg, A. Morales, T. Asfour, and D. Kragic, "Data-driven grasp synthesis - a survey," IEEE Transactions on Robotics, vol. 30, no. 2, pp. 289-309, 2014

[11] K. Hang, J. A. Stork, and D. Kragic, "Hierarchical fingertip space for multi-fingered precision grasping," in Proc. IEEE/RSJ Int. Conf. Intelligent Robots and Systems, 2014.

[12] A. Bicchi and V. Kumar, "Robotic grasping and contact: A review," in Proc. IEEE Int. Conf. Robotics and Automation, 2000.

[13] J. Aleotti and S. Caselli, "A 3d shape segmentation approach for robot grasping by parts," IEEE RAS, vol. 60(3), pp. 358-366, 2012.

[14] M. Przybylski, T. Asfour, and R. Dillmann, "Planning grasps for robotic hands using a novel object representation based on the medial axis transform," in Proc. IEEE/RSJ Int. Conf. Intelligent Robots and Systems, 2011.

[15] N. Vahrenkamp, M. Przybylski, T. Asfour, and R. Dillmann, "Bimanual grasp planning," in Proc. IEEE-RAS Int. Conf. Humanoid Robots, 2011.

[16] K. Huebner, "BADGrA toolbox for box-based approximation, decomposition and GRasping," Robotics and Autonomous Systems, vol. 60, no. 3 , pp. 367 - 376, 2012

[17] R. Pelossof, A. Miller, P. Allen, and T. Jebara, "An svm learning approach to robotic grasping," in Proc. IEEE Int. Conf. Robotics and Automation, 2004

[18] G. Biegelbauer and M. Vincze, "Efficient 3d object detection by fitting superquadrics to range image data for robot's object manipulation," in Proc. IEEE Int. Conf. Robotics and Automation, 2007.

[19] T. T. Cocias, S. M. Grigorescu, and F. Moldoveanu, "Multiplesuperquadrics based object surface estimation for grasping in service robotics," in Optimization of Electrical and Electronic Equipment (OPTIM), 2012, pp. 1471-1477.

[20] D. Berenson, R. Diankov, K. Nishiwaki, S. Kagami, and J. Kuffner, "Grasp planning in complex scenes," in Proc. IEEE-RAS Int. Conf. Humanoid Robots, 2007.

[21] J. A. Stork, F. T. Pokorny, and D. Kragic, "Integrated motion and clasp planning with virtual linking," in Proc. IEEE/RSJ Int. Conf. Intelligent Robots and Systems, 2013.

[22] F. T. Pokorny, J. A. Stork, and D. Kragic, "Grasping objects with holes: A topological approach," in Proc. IEEE Int. Conf. Robotics and Automation, 2013

[23] C. Borst, M. Fischer, and G. Hirzinger, "Grasping the dice by dicing the grasp," in Proc. IEEE/RSJ Int. Conf. Intelligent Robots and Systems, 2003.

[24] B. Mirtich and J. Canny, "Easily computable optimum grasps in 2-d and 3-d," in Proc. IEEE Int. Conf. Robotics and Automation, 1994.

[25] J.-P. Saut and D. Sidobre, "Efficient models for grasp planning with a multi-fingered hand," Robotics and Autonomous Systems, vol. 60, no. 3, pp. $347-357,2012$.

[26] C. Rosales, J. Porta, and L. Ros, "Grasp optimization under specific contact constraints," IEEE Transactions on Robotics, vol. 29, no. 3, pp. 746-757, June 2013.

[27] I. Gori, U. Pattacini, V. Tikhanoff, and G. Metta, "Three-finger precision grasp on incomplete 3d point clouds," in Proc. IEEE Int. Conf. Robotics and Automation, 2014.

[28] C. Ferrari and J. Canny, "Planning optimal grasps," in Proc. IEEE Int. Conf. Robotics and Automation, 1992.

[29] C. Rosales, J. Porta, R. Suarez, and L. Ros, "Finding all valid hand configurations for a given precision grasp," in Proc. IEEE Int. Conf. Robotics and Automation, 2008.

[30] K. Hang, F. T. Pokorny, and D. Kragic, "Friction coefficients and grasp synthesis," in Proc. IEEE/RSJ Int. Conf. Intelligent Robots and Systems, 2013.

[31] M. Roa and R. Suarez, "Computation of independent contact regions for grasping 3-d objects," IEEE Transactions on Robotics, vol. 25, no. 4, pp. 839-850, 2009.

[32] K. Hertkorn, M. Roa, and B. Ch, "Planning in-hand object manipulation with multifingered hands considering task constraints," in Proc. IEEE Int. Conf. Robotics and Automation, 2013.

[33] C. Borst, M. Fischer, and G. Hirzinger, "Grasp planning: how to choose a suitable task wrench space," in Proc. IEEE Int. Conf. Robotics and Automation, 2004 
[34] R. Platt, A. H. Fagg, and R. A. Grupen, "Null-space grasp control: Theory and experiments," IEEE Transactions on Robotics, vol. 26, no. 2, Apr. 2010.

[35] C. Xiong and Y. Xiong, "Neural-network based force planning for multifingered grasp," Robotics and Autonomous Systems, vol. 21, no. 4, pp. $365-375,1997$.

[36] Y. Xia, J. Wang, and L.-M. Fok, "Grasping-force optimization for multifingered robotic hands using a recurrent neural network," IEEE Transactions on Robotics and Automation, vol. 20, no. 3, pp. 549-554, June 2004.

[37] L. Han and J. Trinkle, "Dexterous manipulation by rolling and finger gaiting," in Proc. IEEE Int. Conf. Robotics and Automation, 1998.

[38] J.-P. Saut, A. Sahbani, S. El-Khoury, and V. Perdereau, "Dexterous manipulation planning using probabilistic roadmaps in continuous grasp subspaces," in Proc. IEEE/RSJ Int. Conf. Intelligent Robots and Systems, 2007.

[39] Z. Li, P. Hsu, and S. Sastry, "Grasping and coordinated manipulation by a multifingered robot hand." The International Journal of Robotics Research, vol. 8, no. 4, pp. 33-50, 1989.

[40] T. Yoshikawa and X.-Z. Zheng, "Coordinated dynamic hybrid position/force control for multiple robot manipulators handling one constrained object," The International Journal of Robotics Research, vol. 12, no. 3, pp. 219-230, 1993.

[41] M. Buss, H. Hashimoto, and J. Moore, "Dextrous hand grasping force optimization," IEEE Transactions on Robotics and Automation, vol. 12, no. 3, pp. 406-418, Jun 1996.

[42] T. Takahashi, T. Tsuboi, T. Kishida, Y. Kawanami, S. Shimizu, M. Iribe, T. Fukushima, and M. Fujita, "Adaptive grasping by multi fingered hand with tactile sensor based on robust force and position control," in Proc. IEEE Int. Conf. Robotics and Automation, 2008.

[43] S. A. Schneider and R. H. Cannon, "Object impedance control for cooperative manipulation: theory and experimental results," IEEE Transactions on Robotics and Automation, vol. 8, no. 3, pp. 383-394, 1992.

[44] H. Liu and G. Hirzinger, "Cartesian impedance control for the DLR hand," in Proc. IEEE/RSJ Int. Conf. Intelligent Robots and Systems, 1999.

[45] K. Tahara, K. Maruta, A. Kawamura, and M. Yamamoto, "Externally sensorless dynamic regrasping and manipulation by a triple-fingered robotic hand with torsional fingertip joints," in Proc. IEEE Int. Conf. Robotics and Automation, 2012.

[46] M. Li, H. Yin, K. Tahara, and A. Billard, "Learning object-level impedance control for robust grasping and dexterous manipulation," in Proc. IEEE Int. Conf. Robotics and Automation, 2014.

[47] K. Hsiao, S. Chitta, M. Ciocarlie, and E. Jones, "Contact-reactive grasping of objects with partial shape information," in Proc. IEEE/RSJ Int. Conf. Intelligent Robots and Systems, 2010.

[48] K. Hsiao, L. Kaelbling, and T. Lozano-Perez, "Task-driven tactile exploration," in Proceedings of Robotics: Science and Systems, 2010.

[49] A. Morales, M. Prats, P. Sanz, and A. P. Pobil, "An Experiment in the Use of Manipulation Primitives and Tactile Perception for Reactive Grasping," in Robotics: Science and Systems - Robot Manipulation: Sensing and Adapting to the Real World, 2007.

[50] C. Zito, M. Kopicki, R. Stolkin, C. Borst, F. Schmidt, M. Roa, and J. Wyatt, "Sequential trajectory re-planning with tactile information gain for dexterous grasping under object-pose uncertainty," in Proc. IEEE/RSJ Int. Conf. Intelligent Robots and Systems, Nov 2013.

[51] H. Dang and P. K. Allen, "Grasp adjustment on novel objects using tactile experience from similar local geometry." in Proc. IEEE/RSJ Int. Conf. Intelligent Robots and Systems, 2013.

[52] H. Dang, J. Weisz, and P. Allen, "Blind grasping: Stable robotic grasping using tactile feedback and hand kinematics," in Proc. IEEE Int. Conf. Robotics and Automation, 2011.

[53] Y. Bekiroglu, J. Laaksonen, J. A. Jorgensen, V. Kyrki, and D. Kragic, "Assessing grasp stability based on learning and haptic data," IEEE Transactions on Robotics, vol. 27, no. 3, pp. 616-629, June 2011.

[54] E. L. Sauser, B. D. Argall, G. Metta, and A. G. Billard, "Iterative learning of grasp adaptation through human corrections," Robotics and Autonomous Systems, 2012.

[55] S. Koziel, D. Ciaurri, and L. Leifsson, "Surrogate-based methods," in Computational Optimization, Methods and Algorithms, ser. Studies in Computational Intelligence. Springer Berlin Heidelberg, 2011, vol. 356, pp. 33-59.

[56] C. Walshaw, "Multilevel refinement for combinatorial optimisation problems," Annals of Operations Research, vol. 131, no. 1-4, pp. 325-372, 2004.
[57] N. Sommer, L. Miao, and A. Billard, "Bimanual compliant tactile exploration for grasping unknown objects," in Proc. IEEE Int. Conf. Robotics and Automation, 2014.

[58] F. T. Pokorny and D. Kragic, "Classical grasp quality evaluation: New theory and algorithms," in Proc. IEEE/RSJ Int. Conf. Intelligent Robots and Systems, 2013.

[59] S. El Khoury, M. Li, and A. Billard, "Bridging the gap: One shot grasp synthesis approach," in Proc. IEEE/RSJ Int. Conf. Intelligent Robots and Systems, Oct 2012, pp. 2027-2034.

[60] B. Huang, S. El-Khoury, M. Li, J. Bryson, and A. Billard, "Learning a real time grasping strategy," in Proc. IEEE Int. Conf. Robotics and Automation, May 2013, pp. 593-600.

[61] K. Hang, J. A. Haustein, M. Li, A. Billard, C. Smith, and D. Kragic, "On the evolution of fingertip grasping manifolds," in Proc. IEEE Int. Conf. Robotics and Automation. IEEE, 2016.

[62] T. Yoshikawa, "Manipulability of robotic mechanisms," The International Journal of Robotics Research, 1985.

[63] L. Guilamo, J. Kuffner, K. Nishiwaki, and S. Kagami, "Efficient prioritized inverse kinematic solutions for redundant manipulators," in Proc. IEEE/RSJ Int. Conf. Intelligent Robots and Systems, 2005.

[64] Y. Aydin and M. Nakajima, "Database guided computer animation of human grasping using forward and inverse kinematics." Computers \& Graphics, vol. 23, pp. 145-154, 1999.

[65] M. Powell, "A direct search optimization method that models the objective and constraint functions by linear interpolation," in Advances in Optimization and Numerical Analysis, ser. Mathematics and Its Applications. Springer Netherlands, 1994, vol. 275, pp. 51-67.

[66] T. Wimböck, C. Ott, A. Albu-Schäffer, and G. Hirzinger, "Comparison of object-level grasp controllers for dynamic dexterous manipulation," The International Journal of Robotics Research, vol. 31, no. 1, pp. 3-23, Jan. 2012

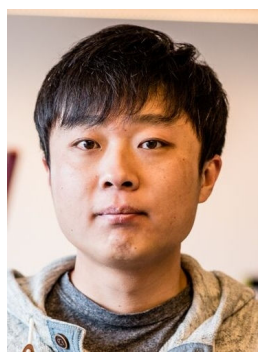

Kaiyu Hang received the B.S. degree in information and communication engineering from Xi'An Jiaotong University, Xi'an, China in 2010 and the M.Sc. degree in communication systems from KTH Royal Institute of Technology, Stockholm, Sweden in 2012. He is currently a Ph.D student at the Centre for Autonomous Systems and the Computer Vision and Active Perception Lab at KTH Royal Institute of Technology, Stockholm, Sweden. His research include robotic grasping, object-hand integrated representation, optimization and manifold learning.

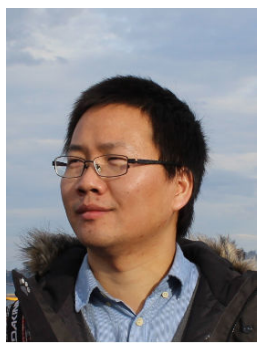

Miao Li received the Bachelor and Masters degree both from the School of Mechanical Science and Engineering, Huazhong University of Science and Technology(HUST), China, in 2008 and 2011 respectively. From Sep. 2011, he joined thecurrent Department of Mechanical Engineer-ing at cole Polytechnique Fdrale de Lausanne (EPFL) in Switzerland, as a doctoral assistant. His current research interests mainly focus on robust robotic grasping, impedance control of dexterous manipulation using tactile sensing. 


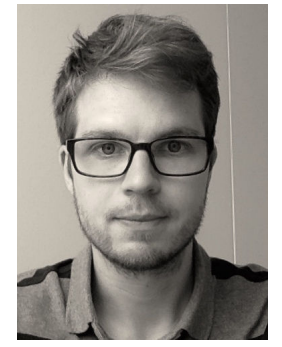

Johannes A. Stork received the B.S. degree in computer science from Albert Ludwigs University of Freiburg, Freiburg, Germany, in 2009 and the M.S. degree in computer science from Albert Ludwigs University of Freiburg, Freiburg, Germany, in 2012. $\mathrm{He}$ is currently pursuing the Ph.D. degree in computer science and robotics at the School of Computer Science and Communication, KTH Royal Institute of Technology, Stockholm, Sweden

From 2009 to 2012, he was a Research Assistant with the Social Robotics Lab, Albert Ludwigs University of Freiburg, Freiburg, Germany. His research interest include representation learning, robotic in-hand manipulation, robotic grab synthesis.

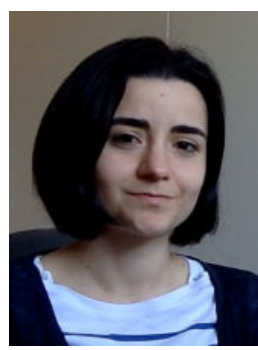

Yasemin Bekiroglu received the M.Sc. degree in computer engineering from Karadeniz Technical University, Trabzon, Turkey, and the M.Sc. degree in applied artificial intelligence from Dalarna University, Dalarna, Sweden. She completed her Ph.D. studies in the Computer Vision and Active Perception Lab (CVAP), in the School of Computer Science and Communication, at the Royal Institute of Technology (KTH), in Stockholm, Sweden, in 2012. Currently, she is a postdoctoral researcher at CVAP. Her research interests include robotics, computer vision and machine learning.

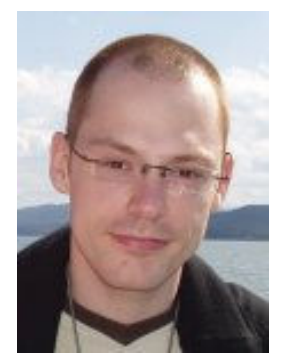

Florian T. Pokorny is a Postdoctoral Researcher at KTH Royal Institute of Technology. He is interested in robotic manipulation and machine learning methods. He received his $\mathrm{PhD}$ in mathematics from the University of Edinburgh and, before that, he completed Part III of the mathematical tripos at the University of Cambridge and obtained a BSc (first class) in Mathematics from the University of Edinburgh.

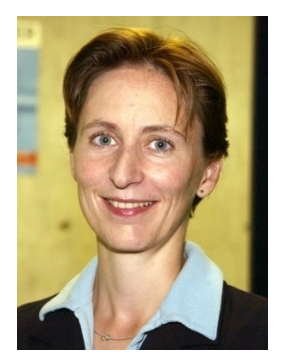

Aude Billard is Professor of Micro and Mechanical Engineering, and the head of the LASA Laboratory at the School of Engineering at the Swiss Federal Institute of Technology in Lausanne. She received a M.Sc. in Physics from EPFL (1995), a MSc. in Knowledge-based Systems (1996) and a Ph.D. in Artificial Intelligence (1998) from the University of Edinburgh. She was the recipient of the Intel Corporation Teaching award, the Swiss National Science Foundation career award in 2002, the Outstanding Young Person in Science and Innovation from the Swiss Chamber of Commerce and the IEEE-RAS Best Reviewer award in 2012. Aude Billard served as an elected member of the Administrative Committee of the IEEE Robotics and Automation society (RAS) for two terms (2006-2008 and 2009-2011) and is the chair of the IEEE-RAS Technical Committee on Humanoid Robotics. Her research interests focus on machine learning tools to support robot learning through human guidance. This extends also to research on complementary topics, including machine vision and its use in human-robot interaction and computational neuroscience to develop models of motor learning in humans.

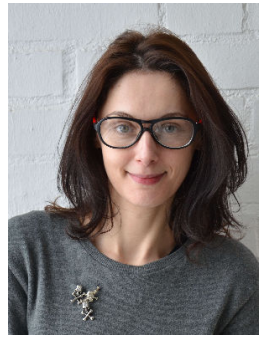

Danica Kragic (SM12) received the M.Sc. degree in mechanical engineering from the Technical University of Rijeka, Rijeka, Croatia, in 1995 and the Ph.D. degree in computer science from the Royal Institute of Technology (KTH), Stockholm, Sweden, in 2001. She is currently a Professor with the School of Computer Science and Communication, KTH. Her research include computer vision, object grasping and manipulation, and humanrobot interaction. Her recent work explores different learning methods for formalizing the models for integrated representation of objects and actions that can be applied on them. This work has demonstrated how robots can achieve scene understanding through active exploration and how full body tracking of humans can be made more efficient.

Dr. Kragic received the 2007 IEEE Robotics and Automation Society Early Academic Career Award. She is a member of the Swedish Royal Academy of Sciences and the Swedish Young Academy. She has chaired the IEEE Robotics and Automation Society (RAS) Technical Committee on Computer and Robot Vision and, since 2009, has served as an IEEE RAS Administrative Committee member. 